\title{
Investigation into factors affecting the mechanical behaviours of a patient- specific vertebral body replacement
}

\author{
Ling Wang', Jianfeng Kang', Lei Shi ${ }^{2}$, Jun $\mathrm{Fu}^{2}$, Dichen $\mathrm{Li}^{1}$, \\ Zheng Guo ${ }^{2}$, Chaozong Liu ${ }^{3}$, Shuangpeng Dong ${ }^{4}$ and Xi Jiang ${ }^{4}$
}

\begin{abstract}
Most vertebral body implants that are currently designed and produced in batches have difficulty meeting the patientspecific demands. Moreover, several complications, including a low fusion rate, subsidence occurrence, and rod displacement, are associated with these implants. This study aims to investigate the effects of patient-specific geometric and clinical parameters on the biomechanics of a vertebral body replacement. A three-dimensional patient-specific vertebral body replacement model was established as the basic model for parametric studies, including the anatomic design of the endplates, tilting angle, thickness, and dislocation of the vertebral body implant. A finite element analysis was applied to determine the stress distribution of the vertebral body implant when under various loading conditions. The model with an anatomical interfacing design generates $75 \%$ less stress concentration compared to a flat design; the peak stress of the model with a tilted angle closely matching the replaced vertebra segment is decreased by $30 \%$; and the thickness close to the cortical bone can offer better bone growth capability and long-term stability. Patient-specific geometrical parameters were found to significantly affect the biomechanics of a vertebral body replacement, and therefore, a design customized especially for the endplates is necessary for better stability and long-term longevity of the prostheses. Regardless of such progress, how to balance the stability of a vertebral body implant and the safety of the peripheral nervous system remains a clinical challenge.
\end{abstract}

\section{Keywords}

Vertebral body replacement, patient-specific design, subsidence, bone graft fusion, finite element analysis

Date received: 28 June 2017; accepted: 2 January 2018

\section{Introduction}

A vertebral body (VB) replacement has been widely accepted for the reconstruction of the spinal support and movement functions for tumour or trauma patients and have shown very high success rates, although certain complications including a subsidence of the vertebral body implant (VBI) due to a lack of permanent fixation to the adjacent tissues still remain. ${ }^{1}$ According to a recent clinical report, the failure rate of titanium alloy in a VBI can reach up to $44 \% .^{2}$ Moreover, the three- and four-month actuarial rates for developing a cage subsidence have been found to be $63.4 \%$ and $70.7 \%$, respectively. ${ }^{3}$ In general, the failure of cage subsidence occurred to the upper vertebrae about $42 \%$ and to the lower vertebrae about $50 \% .^{4}$ The cause of such complications is mainly a mismatch of the VBI to the residual vertebral tissue associated with the stress concentration and loosening. Current commercially available VBIs are produced in batches and show a series of similar patterns with hard-to-fit patient-specific

\footnotetext{
'State Key Laboratory for Manufacturing System Engineering, Xi'an Jiaotong University, Xi'an, China

${ }^{2}$ Department of Orthopaedics, Xijing Hospital, The Fourth Military Medical University, Xi'an, China

${ }^{3}$ John Scale Centre for Biomedical Engineering, University College London, Royal National Orthopaedic Hospital, Stanmore, UK

${ }^{4}$ Tianjin Medical Device Supervision and Testing Center, Tianjin, China
}

\section{Corresponding authors:}

Dichen Li, State Key Laboratory for Manufacturing System Engineering, Xi'an Jiaotong University, No. 99, Yanxiang Road, Yanta District, Xi'an 710054, Shaanxi Province, China.

Email: dcli@mail.xjtu.edu.cn

Zheng Guo, Department of Orthopaedics, Xijing Hospital, The Fourth Military Medical University, No. 15, Changle West Road, Xi'an 710032, Shaanxi Province, China.

Email: guozheng@fmmu.edu.cn 
geometrical characteristics such as the height, tilting angle, and surface curvature at both ends. ${ }^{5}$ Therefore, the customized design of the prosthesis has become necessary, ${ }^{6}$ and with the development of three-dimensional (3D) printing manufacturing technology, it is now feasible to manufacture a prototype with a complex geometry and multi-material properties. ${ }^{1,7}$

To solve the problems of cage subsidence and bone fusion in a VB replacement, the finite element method (FEM) has been widely acknowledged in biomechanical studies on the spine or implant designs ${ }^{8-10}$ because of the advantages of a hypothetical scenario evaluation over a clinical evaluation or mechanical testing. The performances of VB replacements were evaluated based on the range of motion of the fusion segments and the stress distribution of the natural VB and implant. In particular, a smaller range of motion was believed to lead in better stability of the system. ${ }^{11}$ Experimental works have been carried out in vitro to validate the FEM and analyse the mechanical behaviour of an implant. ${ }^{12}$ Previous studies have provided a good methodology for the design of a VBI. Nevertheless, due to the differences in anatomic structure for a natural VB, the influence of various parameters of interbody fusion cages or VBIs on the biomechanical performance has received more attention, including the spatial dimensions of the cervical ring cage, ${ }^{13}$ and the interbody device shape and size. ${ }^{14}$ Clinically, cage subsidence results from numerous factors, and few studies have been conducted to systematically analyse the different effects of patient-specific implant features. ${ }^{7}$ In addition, several studies ${ }^{1,11,15}$ have pointed out that customized VBIs, designed with consideration of the morphology of the vertebrae and the subject-specific Young's modulus distribution, have been proved to effectively minimize the stress shielding and improve the bone graft fusion. However, such designs have brought about certain challenges to surgeons because of the need for perfect implantation techniques, ${ }^{16}$ because any misalignment might result in damage to the peripheral ligaments or muscles. Consequently, the aim of design should be to improve the biomechanical performance through an implementation of patient-specific factors and at the same time to minimize the dependence of the design success on the surgical techniques applied. Therefore, the personalized parameters for the design of VBI were investigated, including the morphology and topology of the end surfaces, the tilting angle, and the wall thickness of the VBI.

\section{Materials and methods}

To investigate the effects of the above-mentioned patient-specific parameters on the biomechanical performance of a VB replacement system with respect to achieving anti-subsidence and long-term stability. Patient-specific models for a VB replacement were built in situ using a VBI, which was designed according to

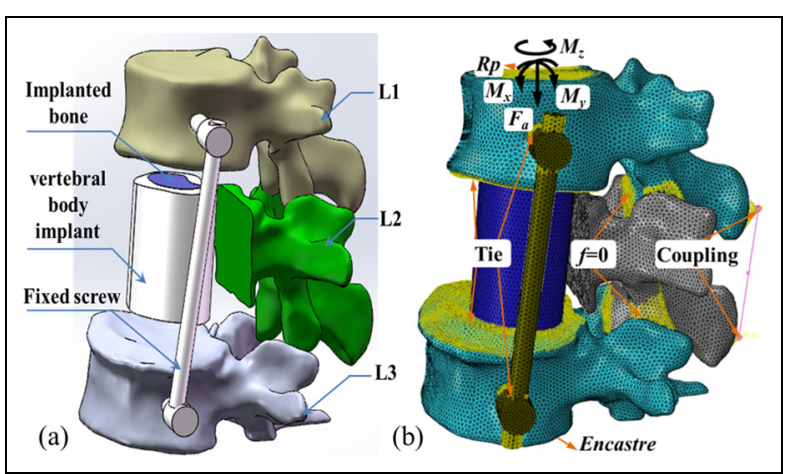

Figure I. (a) The assembly of the LI-L3 spinal segments with vertebral body implanted in and (b) the finite element model configurations of the system showing boundary conditions and loading ( $R p$ denotes for reference point, $M x / M y / M z$ stands for moments along $x / y / z$ axes individually, $F a$ is the loading along axial direction, $f$ represents friction coefficient).

the specific parameters. A finite element analysis was implemented for all of the models to predict the stress distribution in the VBI, as well as the bone grafting, and quantitative studies were carried out for comparison purposes.

\section{Model of a VB replacement}

A model of a VB replacement, based on a real-world clinical scenario, is composed of a natural VB, a designed VBI, and a fixed screw system. A 3D solid model of a VB (L1-L3) was reconstructed from computed tomography (CT) images of a patient using Mimics (Version 16.0, Materialise, Inc., Leuven, Belgium) and smoothed using Geomagic software (Version 2012, Geomagic, Inc., North Carolina, USA), and the whole geometry was then imported into SolidWorks (Versions 2014, Dassault Systemes, Massachusetts, USA), where a portion of the L2 body simulating a diseased area was cut under the instructions of a surgeon. The fixed screw system includes two screws $(\varnothing 5 \times 38 \mathrm{~mm})$ inserted into the middle of the L1 and L3 bodies, respectively, both of which were joined together with a single rod $(\varnothing 4.5 \mathrm{~mm})$. The model of the VBI was constructed using Mimics, and its dimensions were determined based on the reconstructed model of the VB of a patient. The cross-section had a kidney-like shape as suggested by the surgeon, and the area was set to $30 \%-40 \%$ of the area of the connected endplates for better bone integration. ${ }^{17,18}$ The implant was designed as a hollow tube for bone-grafting purposes. The complete model was assembled using SolidWorks, as shown in Figure 1.

The components of each model were meshed using Hypermesh (Version 12.0, Altair Engineering, Inc., USA) and imported into Abaqus (Version 6.14, Abaqus, Inc., USA) for a finite element analysis. To achieve a natural $\mathrm{VB}$, the cancellous part was meshed using a tetrahedron element (C3D4), the cortical part and the cartilage were meshed using a triangular prism 
Table I. Material properties for each component.

\begin{tabular}{lllllc}
\hline Materials & $\begin{array}{l}\text { Elastic modulus } \\
(\mathrm{MPa})\end{array}$ & $\begin{array}{l}\text { Poisson's } \\
\text { ratio }\end{array}$ & Materials & $\begin{array}{l}\text { Elastic modulus } \\
(\mathrm{MPa})\end{array}$ & $\begin{array}{l}\text { Stiffness } \\
(\mathrm{N} / \mathrm{mm})\end{array}$ \\
\hline Cortical bone & 12,000 & 0.3 & Interspinous ligament & 11.6 & 20.17 \\
Cancellous bone & 100 & 0.2 & Ligamentum flavum & 19.5 & 410 \\
Posterior structures & 3500 & 0.25 & Transverse ligament & 58.7 & 0.833 \\
Artificial vertebrae body & 3800 & 0.45 & Posterior longitudinal ligament & 20 & 82.2 \\
Articular cartilage & 10 & 0.3 & Supraspinous ligament & 15 & 55 \\
Pedicle screw system & 110,000 & 0.34 & Capsular ligament & 32.9 & 131.7 \\
Implanted bone & 1850 & 0.3 & & & \\
\hline
\end{tabular}

Table 2. Loading for various daily activities.

\begin{tabular}{lllll}
\hline Loads & Motion types & & & \\
\cline { 2 - 5 } & Standing & Flexion/extension & Left/right lateral bending (LLB/RLB) & Axial rotation \\
\hline Force and moment & $\mathrm{F}=400 \mathrm{~N}$ & $M_{x}=10 \mathrm{~N} \mathrm{~m}$ & $M_{y}=10 \mathrm{Nm}$ & $M_{z}=10 \mathrm{Nm}$ \\
\hline
\end{tabular}

LLB: left lateral bending; RLB: right lateral bending.

element (C4D6), and the rest of the artificial VB and bone graft were meshed using a tetrahedron element. For stability, the relative ligaments were all integrated into the model using nonlinear springs to define the tension-only and incompressible behaviours. ${ }^{19,20}$ Overall, 425,377 elements were included for a complete VB replacement model. The material properties are summarized in Table $1 .^{20,21}$

An interaction property, 'Tie', was applied to define the entire contact surface with the exception of the facet joint. Considering the function of the cartilage, the contact property among the facet joints of the different spinal segments was set to frictionless. All ligaments were represented by a nonlinear spring and built using reference-point coupling according to the anatomic position, mean length, and cross-section. The remaining materials were assumed to be homogeneous, isotropic, and linear elastic. The inferior endplate surface of L3 was constrained within all degrees of freedom. Four loading conditions were applied: standing, flexion, lateral bending (LB), and axial rotation. The corresponding loading magnitudes are summarized in Table $2 .{ }^{22}$ Simulations were then carried out to analyse the stress and strain distributions within the VB, and the interfaces.

\section{Parametrical studies}

In this study, as shown in Figure 2, the key geometrical features and clinical parameters mainly include the morphology of the end surface, the wall thickness, the tilting angle, and the implant position and were investigated using the FEM. Due to the morphology of the end surface for the VBI, which significantly affects the load delivery and stress distribution, two models with one flat and one anatomical surfaces at both ends were analysed. Because a larger implant thickness will reduce the volume of the bone graft, whereas a smaller thickness will decrease the strength of the prosthesis, a wall thickness of $2-3 \mathrm{~mm}$ is usually applied to a commercial VBI. ${ }^{23}$ In this study, five models with five levels of wall thickness between 2 and $4 \mathrm{~mm}$ were considered at $0.5 \mathrm{~mm}$ intervals. Due to the convex forward state of the natural lumbar segments, the influence of the physiological curvature should be taken into account in the VBI design. The tilting angle $\gamma$ was used to represent the physiological curvature of the lumbar in the sagittal plane and was defined as the projection of the angle between the global vertical axis and a line passing through the centres of both the L1 and L3 endplates, also in the sagittal plane. Statistical data ${ }^{24}$ show that the tilting angle normally falls within the range of $0^{\circ}-9^{\circ}$ for the $\mathrm{L} 2$ segment in the human spine; therefore, four designs of $\gamma$ to cover the span of $0^{\circ}-9^{\circ}$ at $3^{\circ}$ intervals were set up for the present study; therefore, four models of various tilting angles were generated. For safety reasons, the VB is normally positioned in the central region rather than at a posterior location to avoid further damage to the peripheral nerve system. To better understand the effects of implant positions, five models corresponding to four locations of the VB were generated, that is, at $3 \mathrm{~mm}$ from the central axis of the L2 medially, laterally, anteriorly, and posteriorly. In total, 16 models were created and studied.

FEM was applied to analyse the influences of the above-mentioned parameters on the stress distribution of the VBI, its adjacent endplates, and the bone graft. Subsequently, the predicted stresses, strain, and percentage of contact area $\left(P_{\text {eca }}\right)$ were employed to determine the characteristics of the implant subsidence and bone ingrowth. 


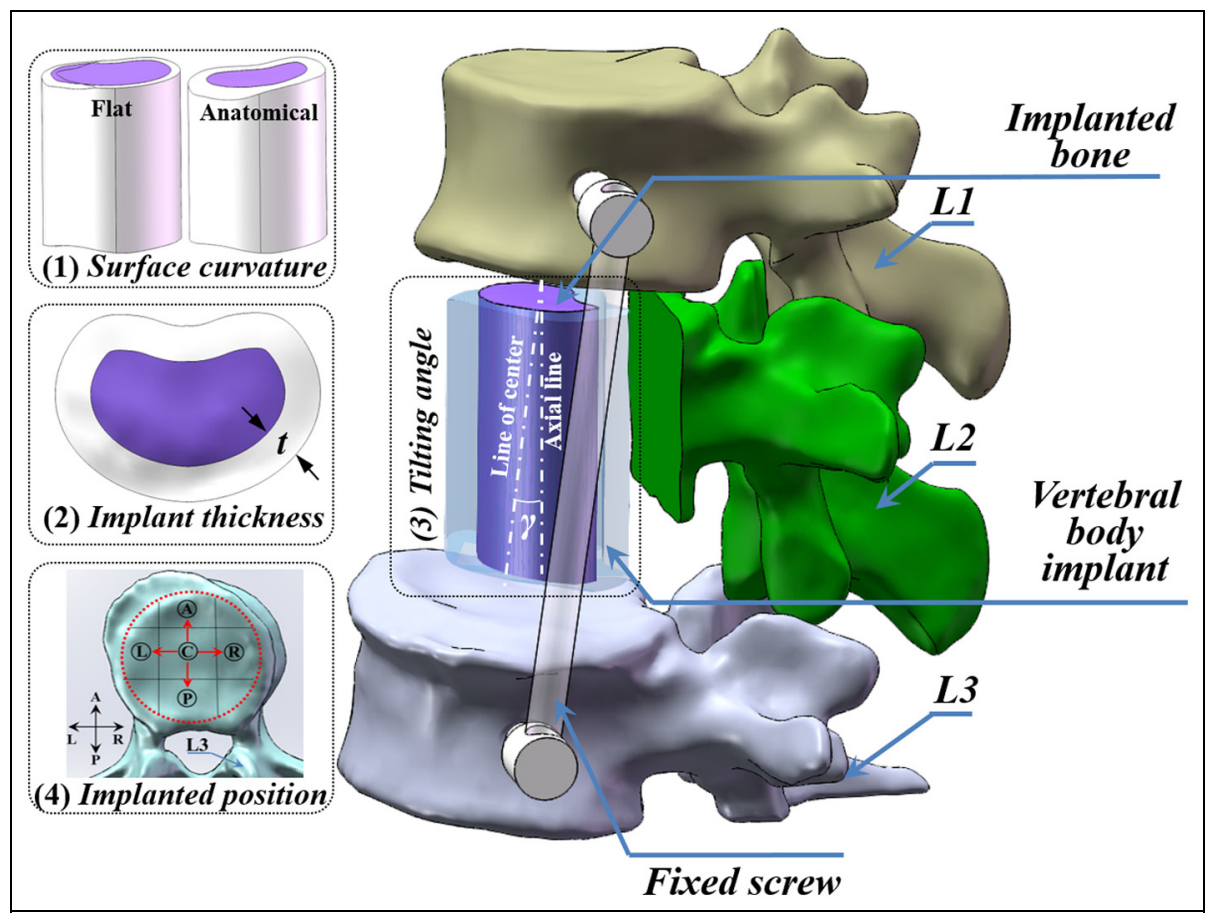

Figure 2. The illustration of investigated factors: (I) flat against anatomical surface configuration, (2) implant wall thickness, (3) tilting angles with respect to the neutral axis and (4) implanted position with $(A)$ stands for anterior, $(\mathbb{P}$ stands for posterior, (L) and (B) stand for left and the right, respectively.

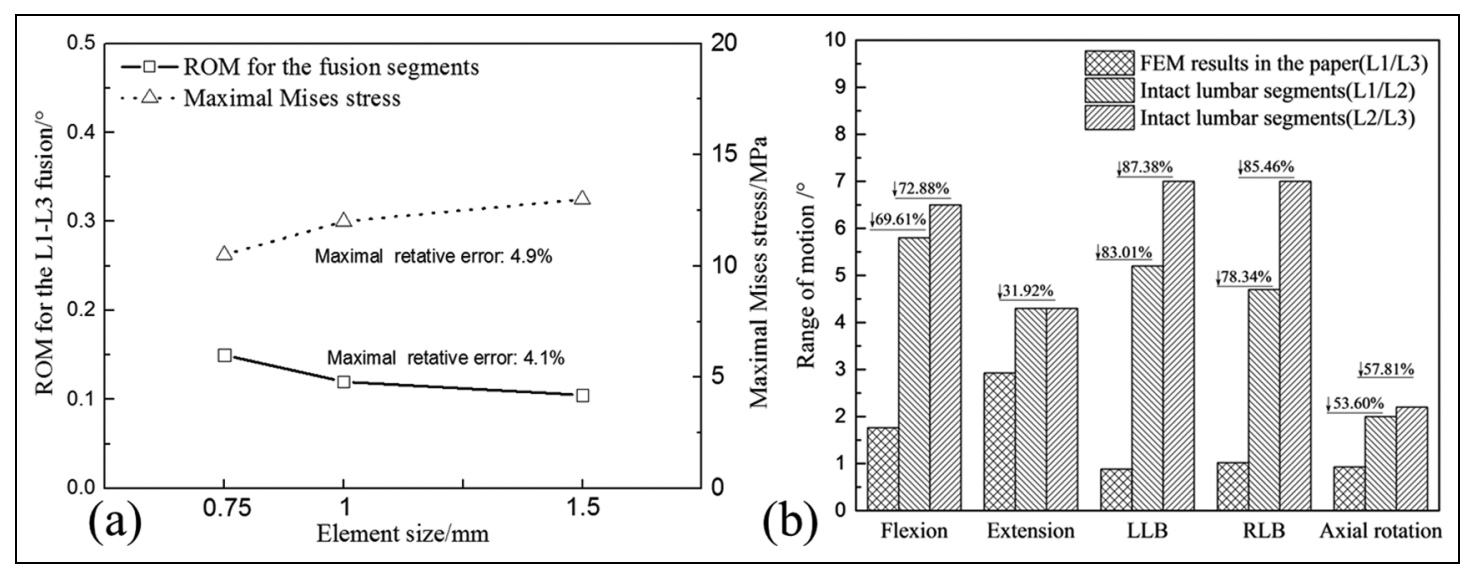

Figure 3. (a) Results of meshing sensitivity study in terms of range of motion (ROM) of the fusion body and von Mises stress within the fusion body and (b) results of ROM for the two interfaces adjacent of the fusion body derived this study compared to those from the literature. ${ }^{12}$

LLB: left lateral bending; RLB: right lateral bending.

A mesh sensitivity study was carried out by employing various element sizes of $0.75,1$, and $1.5 \mathrm{~mm}$ and less than $5 \%$ difference was predicted in terms of the maximum Mises stress within the fusion body as well as the range of motion (Figure 3(a)); therefore, $1 \mathrm{~mm}$ was selected as the universal size of the element throughout this study. Moreover, the range of motion of $1^{\circ}-3^{\circ}$ when under various daily activities was also predicted and compared with the values from the literature ${ }^{12}$ for validation purposes (Figure 3(b)). The results indicated that compared to a natural VB, the replacement offers less range of motion for all activities, particularly for LB.

\section{Results}

Simulation results for the surface designs under four loading conditions are summarized in Figure 4 . The results indicate that the maximum von Mises stresses reach up to $71 \mathrm{MPa}$ for a flat-surface design and $34 \mathrm{MPa}$ for an anatomical design, both of which occur 


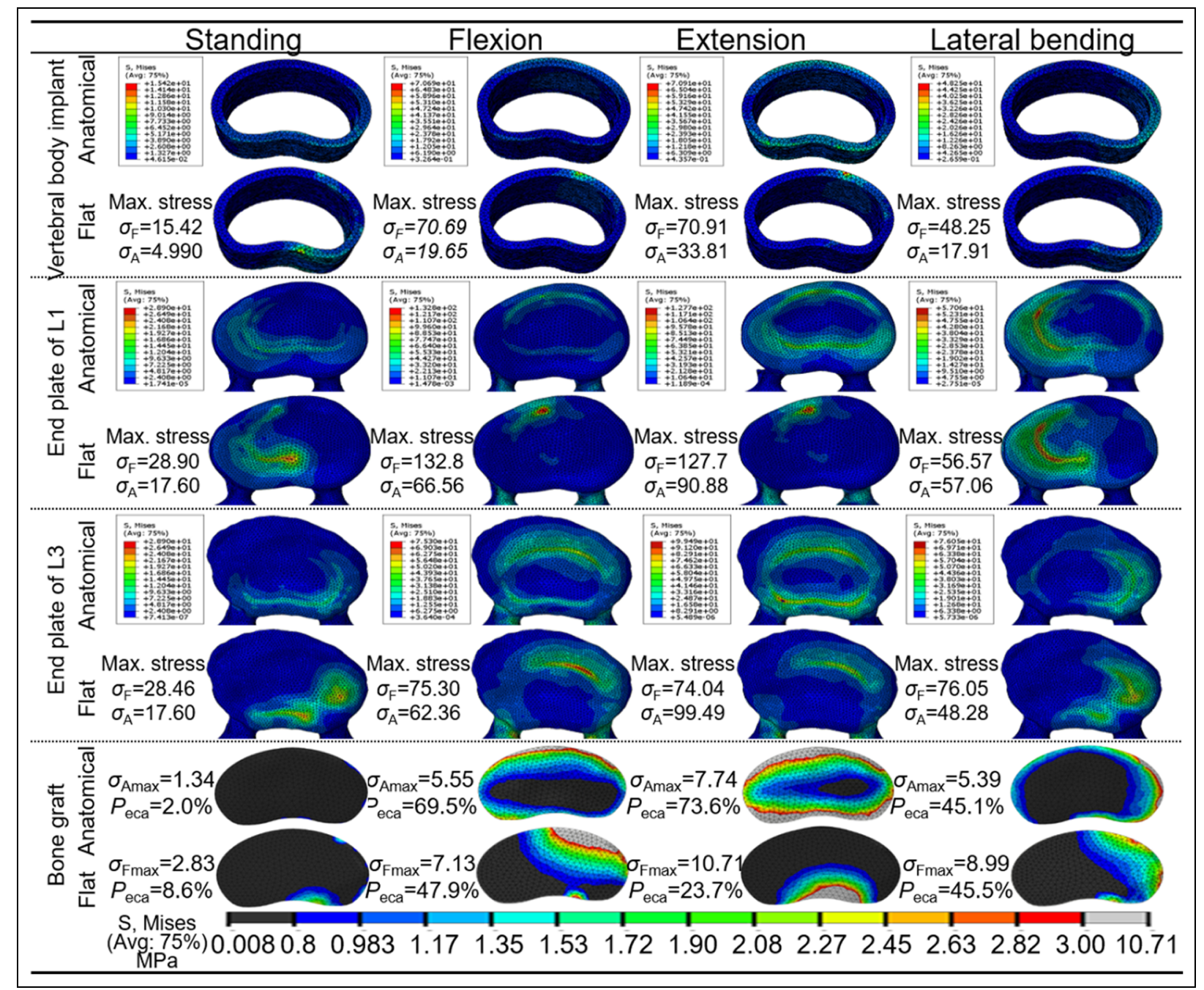

Figure 4. Von Mises stresses predicted for the vertebral body implant, the bottom endplate of $L I$ and the upper endplate of $L 3$, and the bone graft are compared for the flat and anatomical surface designs when under four loading conditions $\left(\sigma_{F}\right.$ and $\sigma_{A}$ stand for maximum von Mises stress for the flat design and the anatomical design; $\sigma_{F \max }$ and $\sigma_{\text {Amax }}$ stand for the maximum von Mises stress on the bone graft for the flat design and the anatomical design; Peca denotes the percentage of effective contact area for contributing to the bone ingrowth).

when under flexion/extension. For the endplates of L1 (only lower endplate of L1 was considered in this study), the maximum von Mises stress was predicted to be above $130 \mathrm{MPa}$ when under flexion for a flat-surface design and $90 \mathrm{MPa}$ for an anatomical design under extension. For the endplates of L3 (only upper endplate of L3 was considered in this study), the maximum von Mises stress was predicted to be $76 \mathrm{MPa}$ when under LB for a flat-surface design and $99 \mathrm{MPa}$ for an anatomical design when under extension.

The simulation results for various thickness designs are shown in Figure 5. The results indicate that an extension motion exerted the maximum stress, whereas a standing motion is correlated with the minimum stress on both the VBI and bone graft. In addition, the predicted von Mises stress in the implant reached the maximum value for a $4 \mathrm{~mm}$ wall thickness, whereas the stresses exerted on the bone graft reached the minimum.

The simulation results for various tilting angles are shown in Figures 6 and 7. A marked discrepancy between the tilting angles can be observed for forward bending and LB to the right. Under forward bending, tilting angles of $0^{\circ}$ and $9^{\circ}$ were predicted to generate maximum von Mises stresses that were approximately $20 \%$ higher than those for tilting angles of $3^{\circ}$ and $6^{\circ}$. For LB to the right, a tilting angle of $0^{\circ}$ was predicted to generate maximum von Mises stresses that were approximately $30 \%$ higher than those for tilting angles of $3^{\circ}, 6^{\circ}$, and $9^{\circ}$. For the remaining loading conditions, no significant differences were observed between the various tilting angle designs. The contour of the stress distribution in Figure 7 indicates that a tilting angle of $6^{\circ}$ provides the largest effective contact area of a bone graft. 


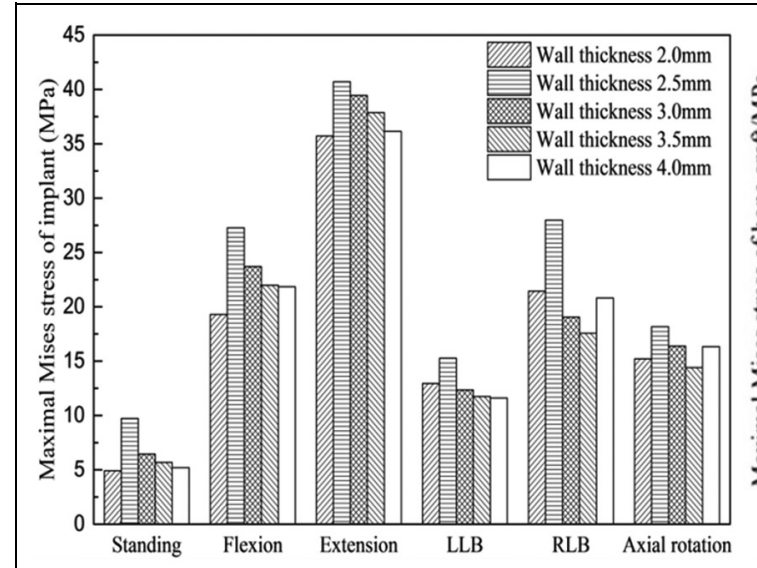

(a)

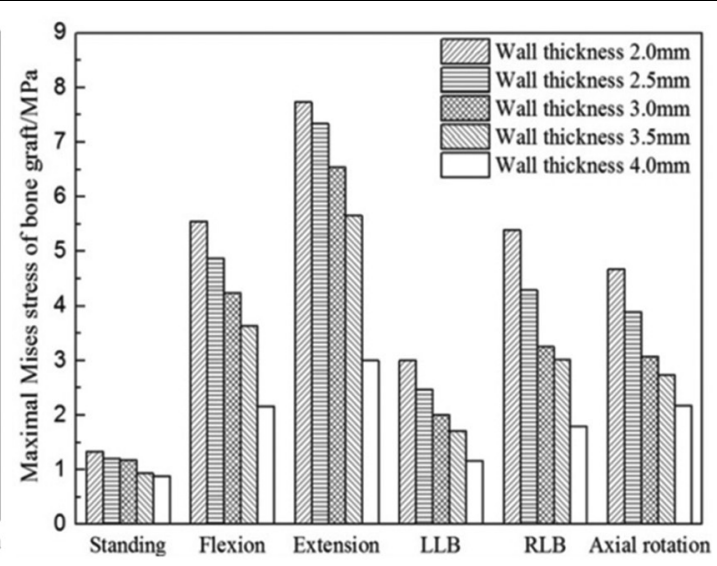

(b)

Figure 5. Von Mises stresses predicted on the vertebral body implant (a) and on the bone graft (b) for models of various wall thickness of vertebral body implant.

LLB: left lateral bending; RLB: right lateral bending.

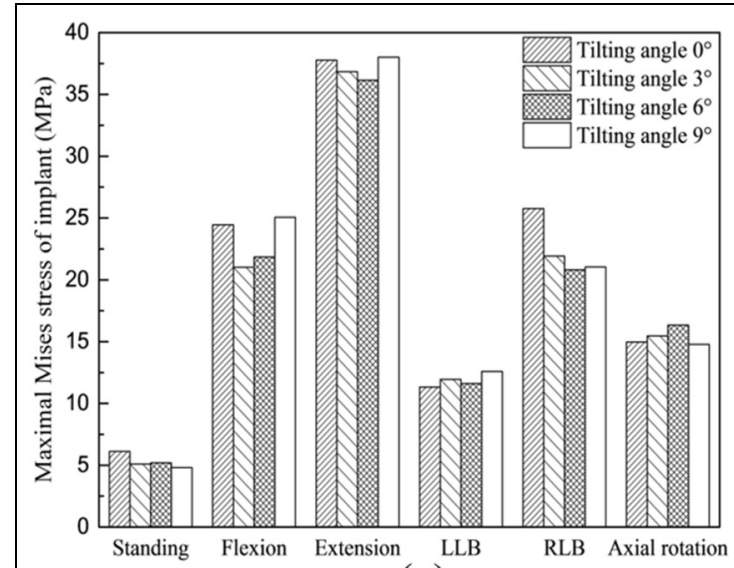

(a)

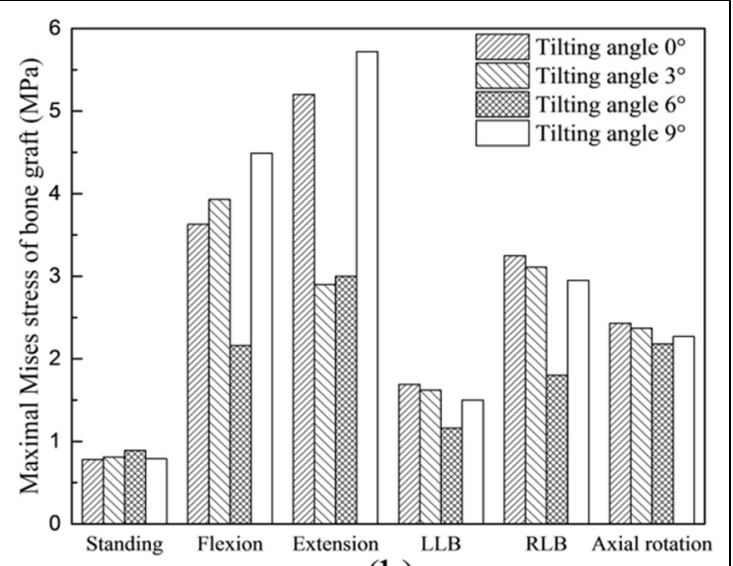

(b)

Figure 6. Von Mises stresses predicted on the vertebral body implant (a) and on the bone graft (b) for models with various tilting angle when under different motions.

LLB: left lateral bending; RLB: right lateral bending.

The simulation results of the implant position (Figures 8 and 9) show that for both the VBI and bone graft, the posterior position offered the lowest predicted maximum stress, and the neutral position offered the second smallest value compared to other positioning scenarios. This difference between the posterior and lateral dislocation scenarios could reach $40 \%$.

\section{Discussion}

Comparable results to those studied here can be achieved from the literature. ${ }^{11,19,25,26}$ Various VBI designs were considered in terms of flat and anatomical cross-sections, various tilting angles, different wall thicknesses, and implant positions, aiming at ranking the effects of such parameters to optimize the design and processing of tailor-made implants. According to the literature, ${ }^{27}$ stress ranging between 0.8 and $3 \mathrm{MPa}$ is believed to stimulate bone-in-growth of the implant. Moreover, cage subsidence is usually defined as a migration of $\geqslant 2 \mathrm{~mm}$ into the adjacent VB. ${ }^{3,28}$

It was found that the range of motion of the VB was restricted and reduced by about $60 \%$ compared to the original spine ${ }^{12}$ because the three adjacent vertebral bodies were almost joined together as a whole. When under various gaits and the associated loading conditions, such as standing up, LB, flexion/extension, and axial rotation, the stresses predicted for the VBI, endplates, and bone graft vary. The smallest stresses were predicted for standing up, while the largest stresses were predicted for flexion/extension motion, which indicates that flexion/extension is the critical motion demanding more strength and stability for the design of VB. Moreover, there is discrepancy between the 


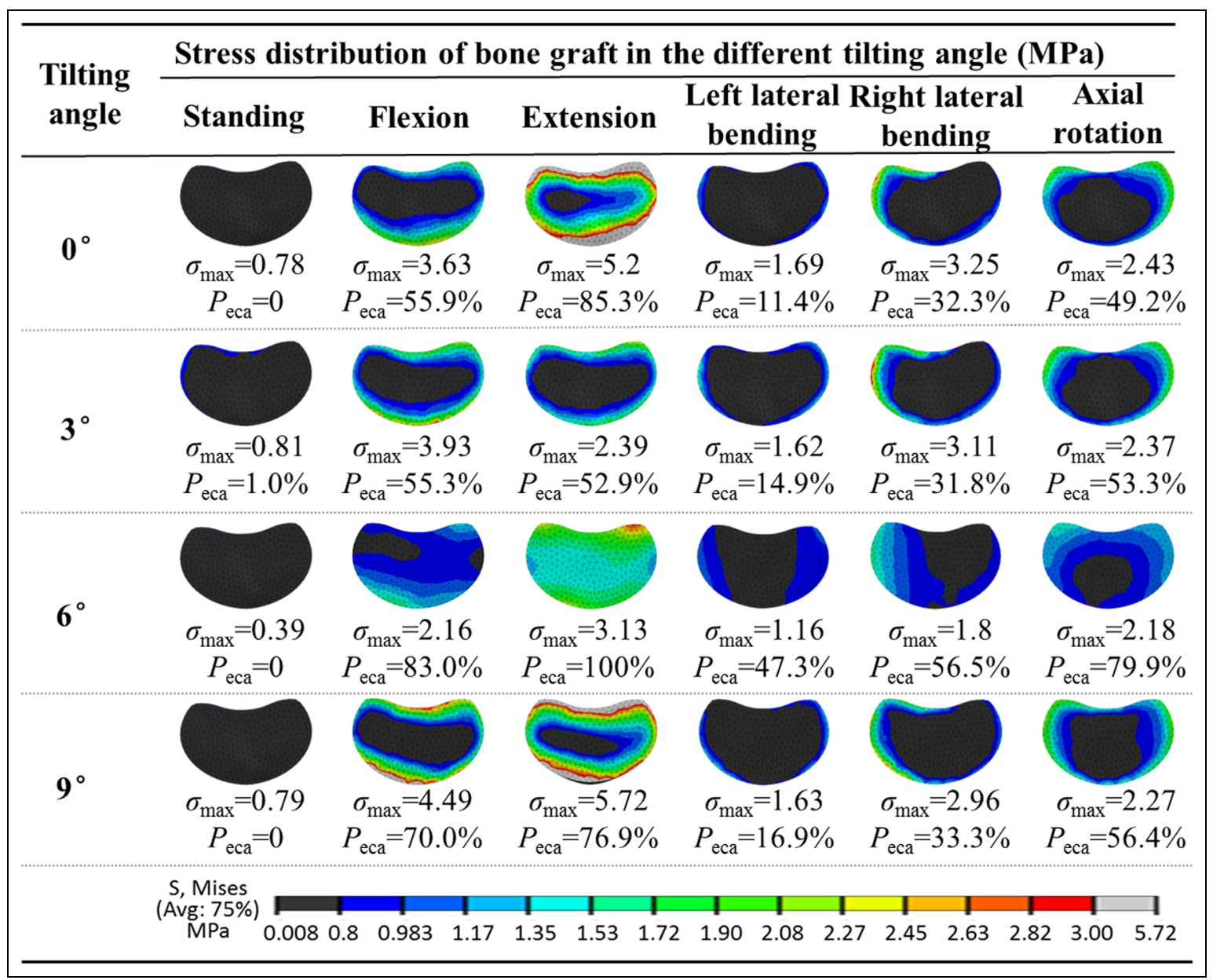

Figure 7. Summary of the contact area and max. Von Mises stresses predicted for the bone grafting for models of different tilting angles when under various motions ( $\sigma_{\max }$ stands for the maximum von Mises stress on the bone graft; Peca denotes the percentage of effective contact area for contributing to the bone ingrowth).

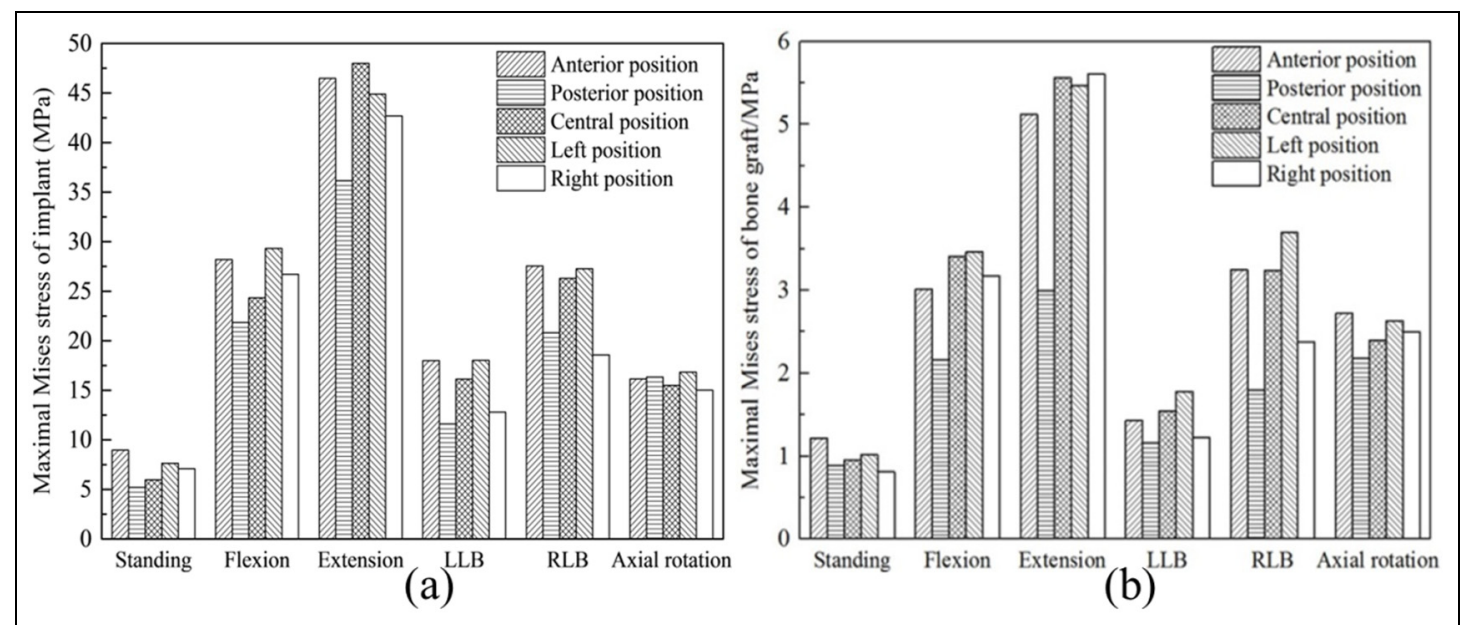

Figure 8. Von Mises stresses predicted for the vertebral body implant (a) and the bone graft (b) for models of various implanting position.

LLB: left lateral bending; RLB: right lateral bending.

stresses predicted for models under left or right lateral motion, which was because of the single-sided fixation system on the left of the VB replacement. The motion of LB to the right was found to generate $20 \%-40 \%$ more stresses compared to that of the LB to the left, and the difference varies with the change of wall thickness; however, this asymmetry characteristics of fixation system was found to have little effect on the 


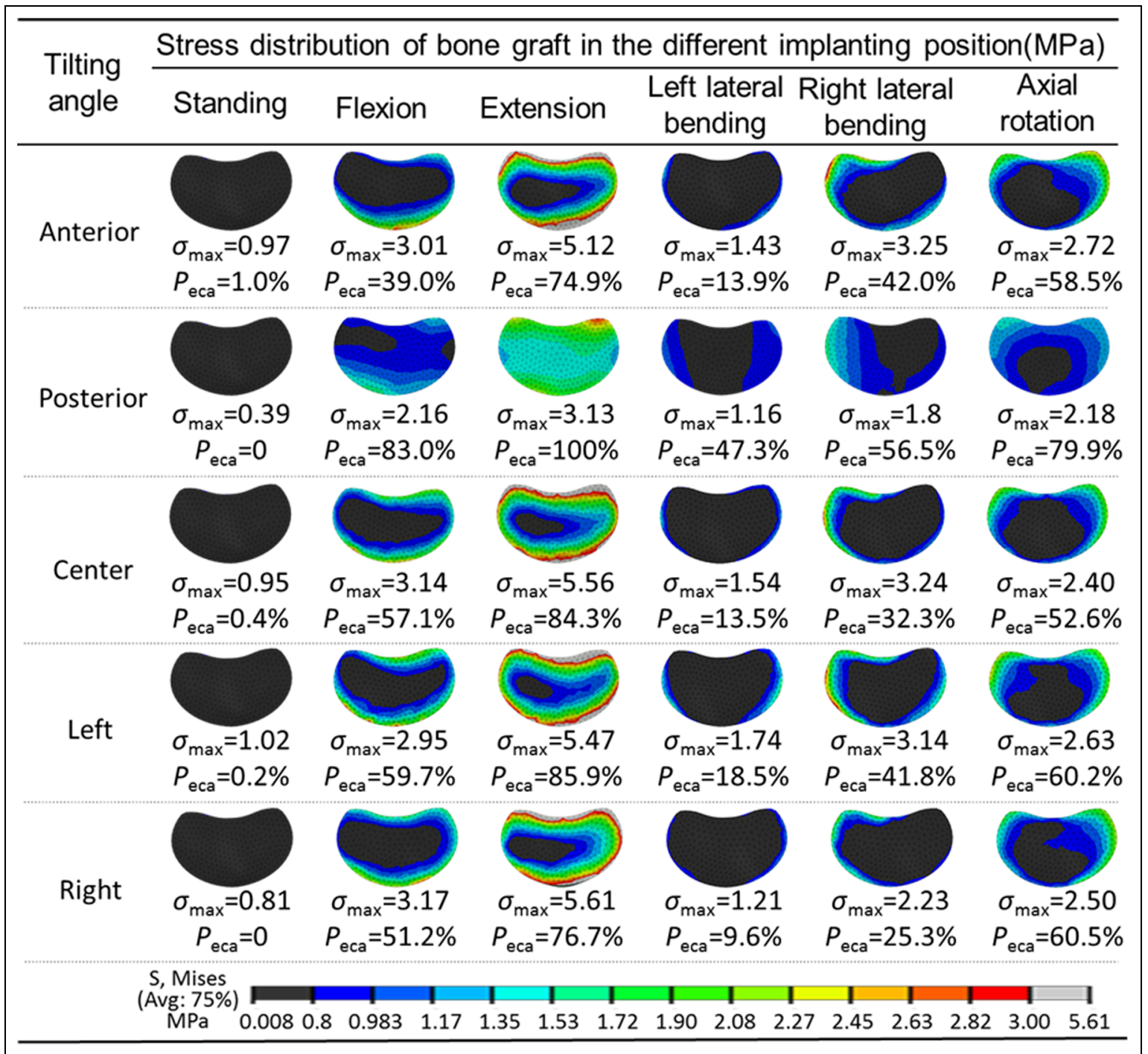

Figure 9. Von Mises stress distribution predicted in bone graft for models of various implanting positions ( $\sigma_{\max }$ stands for the maximum von Mises stress on the bone graft; Peca denotes the percentage of effective contact area for contributing to the bone ingrowth).

stresses generated by motions of axial rotation clockwise or anticlockwise. Additionally, consistent results can be drawn in the predicted range of motion.

Compared with an existing flat-surface design, an anatomical design offers $45 \%$ more effective contact, with a higher uniform stress distribution and less stress concentration. This indicates that an anatomical design can offer better performance of the VBI in terms of reducing the possibility of VBI subsidence in the long run. And this is attributed to better topological fitting at the interfacing surfaces between the implant and endplates. Whatsoever, like the coin has two sides, the anatomical surface design of VBI can also cause large stress concentration if the implant is not positioned properly; therefore, the anatomical design of VBI is very demanding in terms of surgical technique, which obviously hindered its application. ${ }^{7}$

The tilting angle between the central axis and the sagittal plane is subject-specific for an individual VB. The results indicate that the tilting angle does have a markedly effect on the stress on a VB, especially for the extension motion, and models with $0^{\circ}$ or $9^{\circ}$ tilt were found to generate maximum stresses nearly $40 \%$ higher than in a $6^{\circ}$ tilted model. Moreover, the $6^{\circ}$ tilted model was found to generate the largest contact area of the bone graft, which indicates better bone growth potential and better strength of the VB. The explanation for this is that the $6^{\circ}$ tilted model has a tilting angle close to the anatomical tilting angle of the replaced $\mathrm{VB}$, which was $6.8^{\circ}$ according to a clinical image. This makes the anatomical tilting angle an important design parameter.

The VB replacement has been designed in tubular shape for hosting allogenic or autogenic bone tissue inside of it to achieve long-term stability. Therefore, the effects of the thickness of the replacement need to be investigated in terms of the stresses generated on both the VBI and the bone graft. This is because both bodies are bearing load during service of the implant; however, the larger stress the VBI bears, the less stress is exerted to the bone graft, which is believed to cause 
stress shielding and bone loosening in long term. ${ }^{29,30}$ Compared with the surface fitting and tilting angle, the wall thickness did not show a significant effect on the stress distribution of the VBI or bone graft. Among the models with series of thicknesses values being investigated in this study, the model with a thickness of $2.5 \mathrm{~mm}$ was found to generate the largest value of the peak stresses on the VBI, which indicates a larger possibility of implant subsidence in long-term effect. ${ }^{28}$ Therefore, the model with a thickness value of $4 \mathrm{~mm}$ offering the smallest peak stresses for the bone graft was believed to benefit more to bone growth at both endplates after the implantation.

The effect of dislocation distance away from the neutral axis along four coordinate axial directions was investigated. There is no marked differences observed between the models of various implant positions with respect to the stress distribution predicted on the VBI. When the stresses generated in the bone graft are examined, the posteriorly dislocated model was found to exert the most evenly distributed stresses compared to the other types of dislocation models, including the neutral model. In general, the capability of achieving anti-subsidence of a VB replacement can be evaluated based on the stress distributions on the two endplates L1 and L3 interfacing with the VBI, ${ }^{31,32}$ particularly the lower interface of L3 because it bears more weight than the upper interface. ${ }^{33}$ Therefore, the results indicate that posteriorly positioning of the VBI might lead to the best anti-subsidence capability, ${ }^{15}$ which can be seen from the lowest and most evenly distributed stresses at the endplate L3, and from the large contact area on the bone graft associated with a better bone graft sacralization. ${ }^{18,27,34}$ However, it could be challenging to push the posterior of the VBI due to the risk of damage to the nervous tissue.

Limitation of the model developed in this study was mainly the lack of experimental validation, to compare the effective stresses and its distribution with those predicted in this study would make this study more convincible and representative. Moreover, only one natural human vertebral model was studied, which did not have an impact in general perspective; whatsoever, the main purposes of this study were to find out the trends of varied contact mechanics along with the parametrical variation. Additionally, the model developed has been simplified especially for the material properties of the natural vertebrae and endplates, which in reality were not isotropic or homogeneous, while within the permit of accuracy.

\section{Conclusion}

Overall, among all the designing features of the VBI, the surface configuration shows the maximum effect on the stress distribution within the VBI, bone graft and both endplates, followed by the effect of the tilting angle, which made the two factors critically important for the design of VBI in terms of strength and stability of the implant. In addition, the wall thickness plays a marked role in the performance of subsidence prevention in the long run. Moreover, surgeons might be advised to position the VBI towards more posteriorly for better stability but with increasing risk of damaging the spinal cord.

\section{Declaration of conflicting interests}

The author(s) declared no potential conflicts of interest with respect to the research, authorship, and/or publication of this article.

\section{Funding}

The author(s) disclosed receipt of the following financial support for the research, authorship, and/or publication of this article: This work was supported by the Fundamental Research Funds for the Central Universities, and the Research Fund for the Doctoral Program of Higher Education of China (RFDP). This work was also supported by the National Key Research and Development Program of China (2017YFC 1104901) and the Social Development Research Project of Shannxi Provice (2016SF-136).

\section{References}

1. De Beer N and Van der Merwe A. Patient-specific intervertebral disc implants using rapid manufacturing technology. Rapid Prototyping $J$ 2013; 19: 126-139.

2. Behrbalk E, Uri O, Parks RM, et al. Fusion and subsidence rate of stand alone anterior lumbar interbody fusion using PEEK cage with recombinant human bone morphogenetic protein-2. Eur Spine J 2013; 22: 2869-2875.

3. Choi JY and Sung KH. Subsidence after anterior lumbar interbody fusion using paired stand-alone rectangular cages. Eur Spine J 2006; 15: 16-22.

4. Kanayama M, Hashimoto T, Shigenobu K, et al. Pitfalls of anterior cervical fusion using titanium mesh and local autograft. J Spinal Disord Tech 2003; 16: 513-518.

5. Van der Houwen EB, Baron P, Veldhuizen AG, et al. Geometry of the intervertebral volume and vertebral endplates of the human spine. Ann Biomed Eng 2010; 38: 33-40.

6. Lian Q, Li D, Jin Z, et al. Patient-specific design and biomechanical evaluation of a novel bipolar femoral hemiknee prosthesis. J Bionic Eng 2014; 11: 259-267.

7. De Beer $\mathrm{N}$ and Scheffer C. Reducing subsidence risk by using rapid manufactured patient-specific intervertebral disc implants. Spine J 2012; 12: 1060-1066.

8. Bahraminasab M, Sahari BB, Edwards KL, et al. Multiobjective design optimization of functionally graded material for the femoral component of a total knee replacement. Mater Design 2014; 53: 159-173.

9. Zhong ZC, Chen $\mathrm{SH}$ and Hung $\mathrm{CH}$. Load- and displacement-controlled finite element analyses on fusion and non-fusion spinal implants. Proc IMechE, Part H: J Engineering in Medicine 2009; 223: 143-157.

10. Wilcox RK. The biomechanical effect of vertebroplasty on the adjacent vertebral body: a finite element study. 
Proc IMechE, Part H: J Engineering in Medicine 2006; 220: $565-572$.

11. Noailly J, Wilke HJ, Planell JA, et al. How does the geometry affect the internal biomechanics of a lumbar spine bi-segment finite element model? Consequences on the validation process. J Biomech 2007; 40: 2414-2425.

12. Yamamoto I, Panjabi MM, Crisco T, et al. Three-dimensional movements of the whole lumbar spine and lumbosacral joint. Spine 1989; 14: 1256-1260.

13. Yang K, Teo EC and Fuss FK. Application of Taguchi method in optimization of cervical ring cage. $J$ Biomech 2007; 40: 3251-3256.

14. Tan JS, Bailey CS, Dvorak MF, et al. Interbody device shape and size are important to strengthen the vertebraimplant interface. Spine 2005; 30: 638-644.

15. Lowe TG, Hashim S, Wilson LA, et al. A biomechanical study of regional endplate strength and cage morphology as it relates to structural interbody support. Spine 2004; 29: 2389-2394.

16. Rohlmann A, Zander T, Bock B, et al. Effect of position and height of a mobile core type artificial disc on the biomechanical behaviour of the lumbar spine. Proc IMechE, Part H: J Engineering in Medicine 2008; 222: 229-239.

17. Pearcy MJ, Evans JH and O'Brien JP. The load bearing capacity of vertebral cancellous bone in interbody fusion of the lumbar spine. Eng Med 1983; 12: 183-184.

18. Closkey RF, Parsons JR, Lee CK, et al. Mechanics of interbody spinal fusion. Analysis of critical bone graft area. Spine 1993; 18: 1011-1015.

19. Wang W, Zhang H, Sadeghipour K, et al. Effect of posterolateral disc replacement on kinematics and stress distribution in the lumbar spine: a finite element study. Med Eng Phys 2013; 35: 357-364.

20. Xiao Z, Wang L, Gong H, et al. Establishment and verification of a non-linear finite element model for human L4-L5 lumbar segment. In: Proceedings of the 2010 3rd international conference on biomedical engineering and informatics, Yantai, China, 16-18 October 2010. New York: IEEE.

21. Zhao C, Wang X, Chen C, et al. Finite element analysis of minimal invasive transforaminal lumbar interbody fusion. Cell Biochem Biophys 2014; 70: 609-613.

22. Erbulut DU, Zafarparandeh I, Hassan CR, et al. Determination of the biomechanical effect of an interspinous process device on implanted and adjacent lumbar spinal segments using a hybrid testing protocol: a finite-element study. J Neurosurg Spine 2015; 23: 200-208.
23. Coe JD. Instrumented transforaminal lumbar interbody fusion with bioabsorbable polymer implants and iliac crest autograft. Neurosurg Focus 2004; 16: E11.

24. Schlösser TPC, Shah SA, Reichard SJ, et al. Differences in early sagittal plane alignment between thoracic and lumbar adolescent idiopathic scoliosis. Spine J 2014; 14: 282-290.

25. Rohlmann A, Zander $\mathrm{T}$ and Bergmann G. Effects of fusion-bone stiffness on the mechanical behavior of the lumbar spine after vertebral body replacement. Clin Biomech 2006; 21: 221-227.

26. Dreischarf M, Shirazi-Adl A, Arjmand N, et al. Estimation of loads on human lumbar spine: a review of in vivo and computational model studies. J Biomech 2016; 49: 833-845.

27. Weinbaum S, Cowin SC and Zeng Y. A model for the excitation of osteocytes by mechanical loading-induced bone fluid shear stresses. J Biomech 1994; 27: 339-360.

28. Corniola MV, Jägersberg M, Stienen MN, et al. Complete cage migration/subsidence into the adjacent vertebral body after posterior lumbar interbody fusion. $J$ Clin Neurosci 2015; 22: 597-598.

29. Chuah HG, Abd Rahim I and Yusof MI. Topology optimisation of spinal interbody cage for reducing stress shielding effect. Comput Methods Biomech Biomed Engin 2010; 13: 319-326.

30. Denoziere $\mathrm{G}$ and $\mathrm{Ku} \mathrm{DN}$. Biomechanical comparison between fusion of two vertebrae and implantation of an artificial intervertebral disc. J Biomech 2006; 39: 766-775.

31. Polikeit A, Ferguson SJ, Nolte LP, et al. The importance of the endplate for interbody cages in the lumbar spine. Eur Spine J 2003; 12: 556-561.

32. Agarwal A, Palepu V, Agarwal AK, et al. Biomechanical evaluation of an endplate-conformed polycaprolactonehydroxyapatite intervertebral fusion graft and its comparison with a typical nonconformed cortical graft. J Biomech Eng 2013; 135: 61005-61009.

33. Chen SH, Chiang MC, Lin JF, et al. Biomechanical comparison of three stand-alone lumbar cages - a threedimensional finite element analysis. BMC Musculoskelet Disord 2013; 14: 281.

34. Pizanis A, Holstein JH, Vossen F, et al. Compression and contact area of anterior strut grafts in spinal instrumentation: a biomechanical study. BMC Musculoskelet Disord 2013; 14: 254. 